\title{
Carrier Components Assignment Method for LTE and LTE-A Systems Based on User Profile and Application
}

\author{
Husnu S. Narman and Mohammed Atiquzzaman \\ School of Computer Science, University of Oklahoma, Norman, OK 73019 \\ Email: \{husnu, atiq\}@ou.edu
}

\begin{abstract}
Increasing number of mobile users accesses large multimedia files (such as high definition audio, video, images, etc.) over the Internet. Therefore, the bandwidth demand for mobile Internet access is getting exponentially larger. To answer users' demand, Carrier Aggregation is proposed in LTE-A. In Carrier Aggregation, multi bands are used and the bands have supported different coverage. Therefore, mobile users can simultaneously connect only one or multi bands. Because of mobility of users, traffic types and assigned channel errors, the best available Carrier Components of each band should be assigned to each user in order to increase quality of services. Several works have been proposed in the literature to address Carrier Components assignment to mobile users by using Channel Quality Indicator, quality of service and traffic types in LTE systems. However, continuously increasing data requests of users forces the operators to manage traffic more intelligently. Therefore, we have proposed a novel Carrier Component assignment method which considers user profiles and traffic types to increase quality of services and experiences getting by mobile users. Results show that the proposed method uses system resources efficiently and can provide improved throughput rate in LTE and LTE-A systems. Our method will help service providers build efficient Carrier Component assignment services through considering user profile and traffic types.
\end{abstract}

Index Terms-LTE, LTE-A, user profile, carrier component assignment, nonreal time traffic, real time traffic, simulation

\section{INTRODUCTION}

Usage of Mobile Devices (MD) (such as tablet, smartphones, etc.) is increasing significantly and the number of MD for 2013 passed one billion and the expected number of MD for 2017 is almost two billions [1]. The report [1] shows that smartphones and tablets will dominate the future personal computer device market. The most notable reason of increasing $\mathrm{MD}$ is that $\mathrm{MD}$ users can reach wide range of applications under different platforms (e.g., GooglePlay, AppStore) [1] by cutting cross time and place restriction [1], [2]. More than hundred billions mobile applications have been downloaded and more than 250 billions applications are expected to be downloaded for 2017 [1].

Increasing number of mobile users [1] accesses large multimedia files (such as high definition audio, video, images, etc.) over the Internet. Therefore, the bandwidth demand for mobile Internet access is getting exponentially larger [3]. To answer users' demand, Carrier Aggregation (CA) is proposed to extend bandwidth and support $1.5 \mathrm{Gbps}$ for uplink and 3 Gbps for downlink peak data rates in LTE-A [4]. In Carrier
Aggregation multi bands are used and the bands have supported different ranges (i.e., here range means coverage area of each band).

Fig. 1 shows the multi-band architecture in mobile networks. In this architecture, the bands have supported different ranges. MD users can simultaneously connect one or multi bands as showed in Fig. 1. Base stations should arrange the number of simultaneous connections for each band because one band can be overflowed while the other band can be idle. Because of

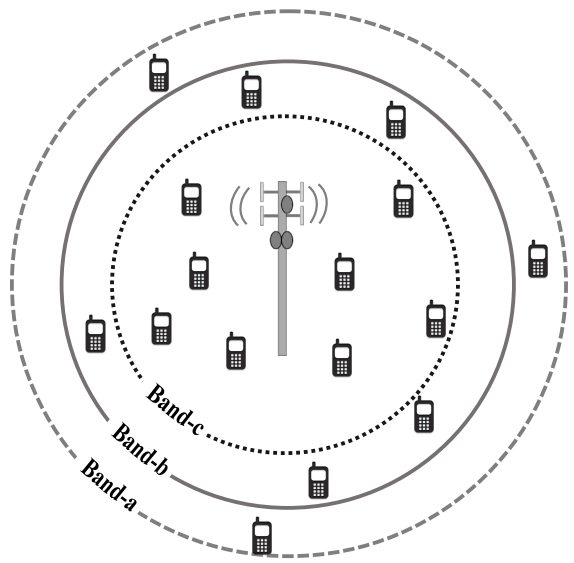

Fig. 1. eNodeB (eNB) with multi bands and several UEs.

mobility of users, traffic types and assigned channel errors, the best available Carrier Components (CCs) of each band should be assigned to each user to increase quality of services [5].

Because of recent improvements in LTE systems, there are several proposed CCs assignment methods [6]-[18] in the literature. In [12], a method is proposed to measure the Channel Quality Indicator (CQI) in LTE-A. In [6]-[9], full or partial feedback is used for CQI to find the best available carrier for each user. In [11], distribution of carriers to users are balanced. In [17], uplink (UL) CA has been proposed by considering a ratio function, traffic type and CQI to increase throughput while sending data from user to eNodeB (eNB). While uplink CCs assignment has bandwidth and power limitation, downlink (DL) CCs assignment has only bandwidth limitation. In [13], [16], service-based methods for CCs assignment are proposed by giving priority for some services while assigning $\mathrm{CCs}$ to users. In [19], CCs are dynamically assigned for each 
user in specified time interval. In addition to the above CCs assignment methods, there exist traditional carrier assignment methods, Least Load (LL) (LL is also called as Round Robin (RR)) and Random (R) [20]. LL and R are well balance traffic loads across different carriers while they ignore Quality of Service (QoS) requirements of each user.

Continuously increasing desired data requests of users forces the operators to manage traffic more intelligently because economic and physical limitations do not allow operators to extend network capacity [21]. Although Load balancing, QoS and CQI methods, as summarized above, have been used to manage traffic and CCs assignment, more advance techniques [21] in addition to these methods will be needed to satisfy users' demands in LTE-A. Therefore, the aim of this work is to propose user profile CCs assignment method in addition to traffic types in order to manage LTE systems more intelligently. None of the above works consider user profiles while assigning CCs to each user. However, not only mobility of each user profile is different but also each user profile needs different QoS from different types of traffic [21]. As illustrated in Table I, bandwidth requirements of each application (Real Time (RT) and Non-real Time (NRT) services) and mobility of each user profiles are different (See Table I for Teenager and Businessman). Therefore, user profiles, in addition to traffic types, can be considered to increase QoS and Quality of Experience (QoE).

The objective of this paper is to increase $\mathrm{QoS}$ and $\mathrm{QoE}$ getting by mobile users by proposing a CCs assignment algorithm which considers user profiles and traffic types. The key contribution of this work are as follows: (i) defining user profiles with respect to traffic types and mobility, (ii) proposing a novel CCs assignment algorithm based on user profiles and traffic types, and (iii) evaluating performance of the proposed method with extensive simulation.

Results show that the proposed CCs assignment method uses system resources efficiently and can provide improved throughput rate in LTE and LTE-A. Therefore, the proposed method and related analysis will help service providers build efficient LTE-A systems architectures which are adaptable to LTE and LTE-A type devices by considering user profile and different types of traffic performances, such as, throughput.

The rest of the paper is organized as follows. In Section II, we explain the system model of LTE-A and user profile with its properties. The proposed method is presented in Section III and simulation environments with parameters are explained in Section IV. In Section V, simulation results are analyzed. Finally, Section VI has the concluding remarks.

\section{System Model ANd User Profile}

In Fig. 1, User Equipments (UEs) are mobile. UEs can connect one band or multi bands simultaneously based on coverages of bands and UEs' positions. UEs can change connected bands to another band in the same eNB if they move from the coverage of one band to the coverage area of another band. For example, when a UE, which is using Band- $b$, enters $B$ and- $c$ range, some of several CCs assignment scenarios for a UE can be as follows (see Fig. 1): (i) the UE may need to use larger bandwidth for services, therefore changing its band to Band-c will increase performance, (ii) mobility of the UE is high, therefore changing its band to Band-c may decrease performance because of low range, (iii) the UE does not need to use larger bandwidth from Band-c, thus no need to update its band, and (iv) the mobility of the UE is high and the UE needs larger bandwidth, therefore it can use both bands. In addition to bands assignment, determining the number of

TABLE I

MOBILE USERS PROFILE

\begin{tabular}{|c|c|c|c|c|c|c|c|}
\hline & & & \multicolumn{5}{|c|}{ User Profile } \\
\hline & & & Teen. & H. wife & B. man & Grad. Stu. & G. parent \\
\hline \multirow{9}{*}{ 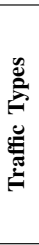 } & \multirow{4}{*}{$\approx$} & Video & V. High & Middle & Low & Medium & Low \\
\hline & & Onl. Game & V. High & Low & Low & Medium & Low \\
\hline & & Movie & V. High & V. High & Low & Medium & Low \\
\hline & & Talk & Low & Medium & High & Medium & V. High \\
\hline & \multirow{3}{*}{$\bar{z}$} & Web & High & Low & V. High & Medium & Low \\
\hline & & Mail & High & Low & V. High & Medium & Low \\
\hline & & SMS & V. High & Medium & Low & Medium & Low \\
\hline & \multirow{2}{*}{$\dot{\tilde{\Xi}}$} & Mobility & Low & Medium & V. High & Low & Low \\
\hline & & Location & Low & Medium & High & Medium & Low \\
\hline
\end{tabular}

required CCs for each UE is significant because of power and QoS efficiency. For example, when a UE can enter an eNB range, some of scenarios to determine the number of CCs for the UE can be as follows: (i) data usage of the UE is small, therefore only one $\mathrm{CC}$ will be enough, (ii) the data usage of the UE is high, therefore, assigning multi CCs will increase performance, and (iii) device type of the UE is not allowed to assign more than one $\mathrm{CC}$, therefore, one $\mathrm{CC}$ will be assigned. Above scenarios show the importance of management of CCs in LTE and LTE-A in order to increase performance.

\section{A. System Model}

Fig. 2 shows system model for a CCs assignment method. There are $n$ number of UEs and each UE can only connect up to $m$ number of CCs. One to two of CCs are primary component carrier (PCC) for DL and UL, and can only be updated during handover [4], but the rest of CCs can be dynamically assigned to each UE in specified time interval [19]. Today, LTE-A system can only support five CCs for each UE in order to provide LTE-A level service [4]. However, assigning

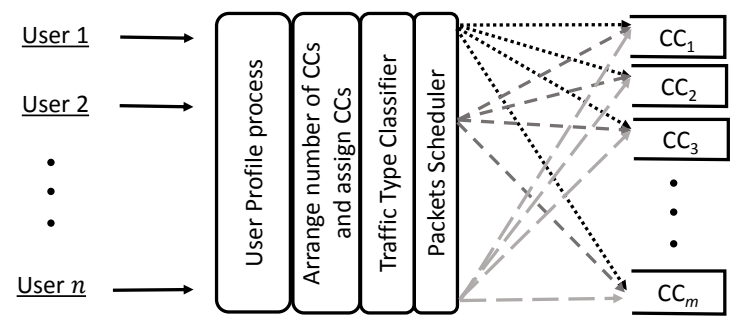

Fig. 2. System Model with $n$ users and $m$ available CCs.

all available CCs to a UE can increase power consumption and interference. Therefore, it is important to have a CCs assignment method, which firstly determines the number of required CCs and band of each CCs for each UE then assign 
them. Determining the number of required $\mathrm{CCs}$ and band of each CCs for each UE does not only decrease power consumption and interference but also increase efficiency of $\mathrm{CCs}$ resources usage. The only way to find required $\mathrm{CCs}$ is to estimate data usage and mobility of UEs (user profiles). Estimating RT and NRT data usage for a UE helps an eNB arrange the number of $\mathrm{CCs}$ and their bandwidth sizes, and estimating mobility of a UE reduces handover overheads and risk of connection lost. In Section II-B, we have demonstrated how to estimate data usage and mobility of each UE based on user profile. It is important to note that the user profile can be used with any existing CCs assignment methods.

\section{B. User Profile Detection Based on Services}

Historical data usage information of each UE plays crucial role to identify user profiles. As shown in Table II, each UE holds Times, Connection Time (Con. T) and Idle Time (Idle $T$.), $R T$ and NRT services data sizes for each eNB. In Table II, Times illustrates how often a UE connects to eNBs, Con. T represents how long a UE keeps connected eNBs and Idle T. gives how long UE connected but not receive any services from previous sessions for each band.

TABLE II

USER PRofile Detection BASED ON ENodeBs

\begin{tabular}{|c|c|c|c|c|c|c|c|}
\hline & \multicolumn{9}{|c|}{ Band-a/Band-b/Band-c } & \multicolumn{2}{c|}{ RT-Services } & \multicolumn{2}{c|}{ NRT-Services } \\
\hline eNB-ID & Times & Con. T. & Idle T. & Video & Game & Web & Mail \\
\hline$I D_{1}$ & $f_{1}$ & $c_{1}$ & $t_{1}$ & $v_{1}$ & $g_{1}$ & $w_{1}$ & $m_{1}$ \\
\hline$I D_{2}$ & $f_{2}$ & $c_{2}$ & $t_{2}$ & $v_{2}$ & $g_{2}$ & $w_{2}$ & $m_{2}$ \\
\hline$I D_{3}$ & $f_{3}$ & $c_{3}$ & $t_{3}$ & $v_{3}$ & $g_{3}$ & $w_{3}$ & $m_{3}$ \\
\hline$I D_{4}$ & $f_{4}$ & $c_{4}$ & $t_{4}$ & $v_{4}$ & $g_{4}$ & $w_{4}$ & $m_{4}$ \\
\hline$I D_{5}$ & $f_{5}$ & $c_{5}$ & $t_{5}$ & $v_{5}$ & $g_{5}$ & $w_{5}$ & $m_{5}$ \\
\hline$I D_{6}$ & $f_{6}$ & $c_{6}$ & $t_{6}$ & $v_{6}$ & $g_{6}$ & $w_{6}$ & $m_{6}$ \\
\hline$I D_{7}$ & $f_{7}$ & $c_{7}$ & $t_{7}$ & $v_{7}$ & $g_{7}$ & $w_{7}$ & $m_{7}$ \\
\hline$I D_{8}$ & $f_{8}$ & $c_{8}$ & $t_{8}$ & $v_{8}$ & $g_{8}$ & $w_{8}$ & $m_{8}$ \\
\hline
\end{tabular}

In order to identify user profile from Table II, some statical analysis such as percentage measurement, can applied. For example, percentage of Connection Time of UE $i$ to eNB $j$ $\left(\Delta C_{j}^{i}\right)$ and percentage of Times of UE $i$ to eNB $j\left(\Delta T_{j}^{i}\right)$ can be simply calculated as follows:

$$
\Delta C_{j}^{i}=100 \times \frac{c_{j}}{\sum_{s=1}^{k} c_{s}} \text { and } \Delta T_{j}^{i}=100 \times \frac{f_{j}}{\sum_{s=1}^{k} f_{s}}
$$

where $k$ is the number of eNBs. Lower $\Delta T_{j}^{i}$ and higher $\Delta C_{j}^{i}$ indicate that UE $i$ spends its more time around eNB $j$ with specified carrier band. On the other hand, higher $\Delta T_{j}^{i}$ and lower $\Delta C_{j}^{i}$ indicate that UE $i$ temporarily requests service from eNB $j$. For example, UE $i$ just uses eNB $j$ while driving home, to work or school.

Data usage of a UE can also be estimated from Table II. For example, percentage of RT services for UE $i$ in eNB $j$ can be simply measured as

$$
\Delta R T_{j}^{i}=100 \times \frac{v_{j}+g_{j}}{\sum_{s=1}^{k}\left(v_{s}+g_{s}\right)}
$$

Like $\Delta R T_{j}^{i}, \Delta N R T_{j}^{i}$ can be obtained. Furthermore, active time percentage of UE $i$ in eNB $j\left(\Delta A T_{j}^{i}\right)$ can be measured as

$$
\Delta A T_{j}^{i}=100 \times \frac{c_{j}-t_{j}}{\sum_{s=1}^{k} c_{s}-\sum_{s=1}^{k} t_{s}}
$$

Similarly, data usage percentage of each service for any eNB ID can be measured as above without classifying RT and NRT services.

In addition to percentage analysis, average analysis can be applied. For example, average connection time $\left(\Theta C_{j}^{i}\right)$, average RT $\left(\Theta R T_{j}^{i}\right)$ and average NRT $\left(\Theta N R T_{j}^{i}\right)$ data usage of UE $i$ in eNB $j$ can be measured per connection as follows:

$$
\Theta C_{j}^{i}=\frac{c_{j}}{f_{j}}, \quad \Theta R T_{j}^{i}=\frac{v_{j}+g_{j}}{f_{j}}, \quad \Theta N R T_{j}^{i}=\frac{w_{j}+m_{j}}{f_{j}}
$$

More average analysis can be used by an eNB to identify a UE profile although no information is available for the eNB in user profile table.

Eqns. (1) - (4) are some of examples which can be used to identify user profiles based on Table II in order to provide services which meet expectation of each UE.

\section{CCS Assignment Method FOR LTE $\backslash$ LTE-A SYSTEM}

Fig. 3 illustrates the proposed CCs assignment method in LTE systems. Simply, the proposed method firstly finds the number of required CCs and bands of CCs, and assigns them to each UE. The proposed method considers three crucial parameters that enable dynamic CCs assignment: (i) UE device

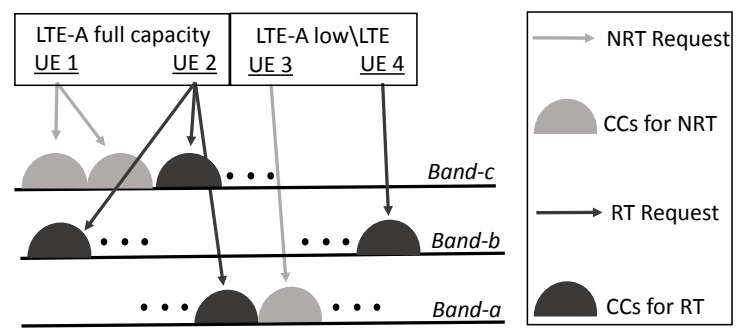

Fig. 3. Illustration of CCs assignment in LTE systems.

capacity in terms of LTE, LTE-A low capacity, and LTEA full capacity, (LTE-A low capacity should be considered because multi-CCs assignment needs more memory and power for processing [5]. Therefore, only one $\mathrm{CC}$ can be assigned for LTE and LTE-A low capacity), (ii) data traffic types of incoming requests (RT or NRT), and (iii) user profiles of UEs.

\section{A. Number of Required CCs for Each UE}

In order to estimate the number of required CCs for UE $i$ in eNB $j$, total and average data usage which obtained from Table II are used. Therefore:

$$
\alpha=\frac{\Theta R T_{j}^{i}}{\sum_{s=1}^{k} \frac{v_{s}+g_{s}}{f_{s}}} \text { and } \beta=\frac{\Theta N R T_{j}^{i}}{\sum_{s=1}^{k} \frac{w_{s}+m_{s}}{f_{s}}}
$$


The number of required CCs for RT traffic $\left(\eta R T_{j}^{i}\right)$ and NRT traffic $\left(\eta N R T_{j}^{i}\right)$ for UE $i$ in eNB $j$ can be obtained by using $\alpha$ and $\beta$ as follows:

$$
\eta R T_{j}^{i}=\left\{\begin{array}{lll}
1 \times C C & \text { if } & \frac{\alpha}{\xi} \leqslant 1 \\
\frac{\alpha}{\xi} \times C C & \text { if } & \frac{\alpha}{\xi} \geqslant 1
\end{array} \text { and } \quad \frac{\alpha}{\xi}+\frac{\beta}{\xi} \leqslant 5\right.
$$

and

$$
\eta N R T_{j}^{i}= \begin{cases}1 \times C C & \text { if } \quad \frac{\beta}{\xi} \leqslant 1 \\ \frac{\beta}{\xi} \times C C & \text { if } \quad \frac{\beta}{\xi} \geqslant 1 \quad \text { and } \quad \frac{\alpha}{\xi}+\frac{\beta}{\xi} \leqslant 5\end{cases}
$$

where $\xi$ is the maximum data rate which a CC can carry for active UEs. $\xi$ can be calculated by considering modulations and the number of UEs which are waiting for services in eNB j. $\alpha / \xi+\beta / \xi \leqslant 5$ because only five CCs will be aggregated in LTE-A. If $\alpha / \xi+\beta / \xi>5$, CCs are divided for RT and NRT services according to rate between $\Theta N R T_{j}^{i}$ and $\Theta R T_{j}^{i}$.

\section{B. CCs Assignment Process}

By using above parameters, proposed CCs assignment method process is as follows: (i) getting info about user device capacity, (ii) finding all available CCs from resources, (iii) measuring the number of UEs waiting for services to find suitable $\mathrm{CCs}$ for each UE, (iv) reserving some $\mathrm{CCs}$ with appropriate bandwidth sizes for NRT and RT services, (v) measuring UE profile metrics by following procedure in Sections II-B and III-A to determine the bands (whether Band- $a$, Band- $b$, Band- $c$ or multi bands) and estimating the number of required $\mathrm{CCs}$ in each band, (vi) assigning the number of required $\mathrm{CCs}$ which are determined based on user profile to each UE (if there are more available CCs in specified bands than the number of required CCs, one of CCs scheduling algorithms such as $\mathrm{R}$ or LL can be used) and (vi) repeating process in time intervals.

\section{Simulation of The System}

We have written discrete event simulation in Matlab by taking into account the mentioned CCs assignment method in Sections II and III.

\section{A. Assumptions for eNBs}

While implementing simulation, it is assumed that there is only one eNB which has three bands to provide service to UEs. The bands are divided as NRT and RT CCs. CCs for NRT and RT services and their sizes and quantities are given in Table III. The sizes and quantities are arranged based on the $800 \mathrm{MHz}, 1.8 \mathrm{GHz}$ and $2.6 \mathrm{GHz}$. To reach required data rate for

TABLE III

NUMbER of $C C s$ WITH BANDWIDTH SIZE IN EACH BANDS

\begin{tabular}{|c|c|c|c|c|c|c|}
\hline & \multicolumn{2}{|c|}{ Band- $a$} & \multicolumn{2}{c|}{ Band- $b$} & \multicolumn{2}{c|}{ Band-c } \\
\hline & Quantity & Size & Quantity & Size & Quantity & Size \\
\hline NRT & $\mathrm{x}$ & $10 \mathrm{MHz}$ & 4 & $10 \mathrm{MHz}$ & 4 & $10 \mathrm{MHz}$ \\
\hline RT & $5-\mathrm{x}$ & $10 \mathrm{MHz}$ & 5 & $20 \mathrm{MHz}$ & 4 & $20 \mathrm{MHz}$ \\
\hline
\end{tabular}

LTE systems, $10 \mathrm{MHz}$ bandwidth is chosen for NRT services and $20 \mathrm{MHz}$ bandwidth is chosen for RT services from Band-b and Band-c, and only $10 \mathrm{MHz}$ bandwidth is chosen for RT and NRT services from Band- $a$ because PCC is generally chosen from a band which has higher range like Band-a. Therefore, the bandwidth size of CCs is kept $10 \mathrm{MHz}$ for Band-a. In addition, bandwidth size of NRT type CCs is $10 \mathrm{MHz}$ because RT traffic data usage is more common than NRT data usage for mobile devices. Size of NRT and RT packets is 512 bytes [22]. Therefore, NRT CCs can carry 10 packets and RT CCs can carry 20 packets simultaneously by considering $25 \%$ percent lower than CCs capacities because of bit and channels errors, 64QAM bit rate with normal cyclic prefix and 2 Physical Downlink Control Channel (PDCCH) symbols.

\section{B. Assumptions for UEs}

There are three types of UEs, LTE, LTE-A low and LTE-A full capacities in the system. 2/3 of UEs can only use one CC but $1 / 3$ of UEs can use multiple CCs. UEs are uniformly distributed in area and UEs can use one or multi bands. $50 \%$ of UEs can move around of the eNB for every iteration in specified time interval. Each UE can only generate one type of traffic (NRT or RT). Packet arrivals follow Poisson distribution and arrival rates of traffic are getting higher when the number of users is increased. Selected Transmission Time Interval (TTI) for a packet is $1 \mathrm{~ms}$. CCs updating time for UEs is $10 \mathrm{~ms}$.

\section{Observation Methodology}

The simulation results in Section V are average of 1000 simulation runs for different UEs size. We observe the impact of light and heavy UEs loads on CCs assignment procedure mentioned in Section III-A by using Random CCs assignment (R). R method is chosen for test cases because of simplicity. There are three possible ways in order to see user profile CCs assignment method effects on $\mathrm{R}$ method. They are: (i) how only data usage estimation based on user profile affects $\mathrm{R}$ method, (ii) how only mobility estimation based on user profile affects $\mathrm{R}$ method, and (iii) how both data usage and mobility estimation affect $\mathrm{R}$ method? In this report, only data usage estimation based on user profile is used with simple mobility estimation in order to show effects of the proposed method on $\mathrm{R}$ method. In mobility estimation, just previously connected bands are used without considering connection time (Cont. T. in Table II)). Shortly, after finding the number of CCs for a UE by estimating data usage, the number of CCs for the UE are chosen from bands which were used previously by the same $\mathrm{UE}$ if the UE is in the same or close to same position.

Random CCs assignment with the static number of $\mathrm{CCs}$ (RSA), Random CCs assignment with the dynamic number of CCs based on perfect user profile estimation (UPR), Random $\mathrm{CCs}$ assignment with the dynamic number of CCs based on user profile estimation for $10 \%$ error $\left(\mathrm{UPR}^{10}\right)$ and Random $\mathrm{CCs}$ assignment with the dynamic number of $\mathrm{CCs}$ based on user profile estimation for $25 \%$ error $\left(\mathrm{UPR}^{25}\right)$ have been analyzed. User profile of each UE for UPR ${ }^{10}$ and $\mathrm{UPR}^{25}$ is obtained by adding $10 \%$ and $25 \%$ errors, respectively, Error means that data usage is estimated based on these above error percentages. For example, a UE data usage rate is $100 \mathrm{MB}$ 


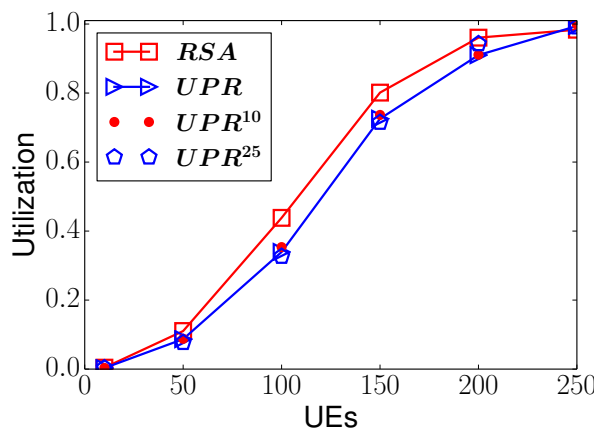

Fig. 4. Utilization of Band-a for RSA and UPRs.

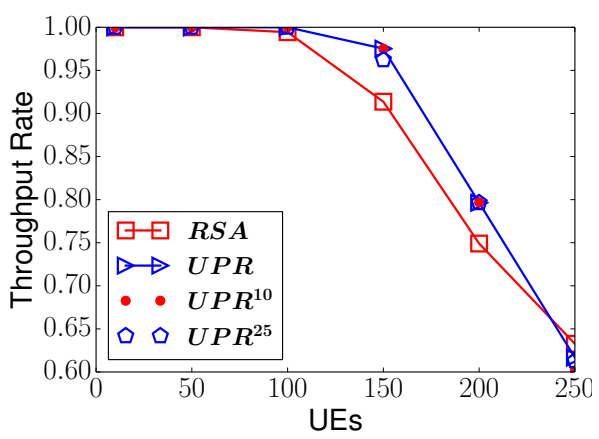

Fig. 7. NRT traffic throughput for RSA and UPRs.

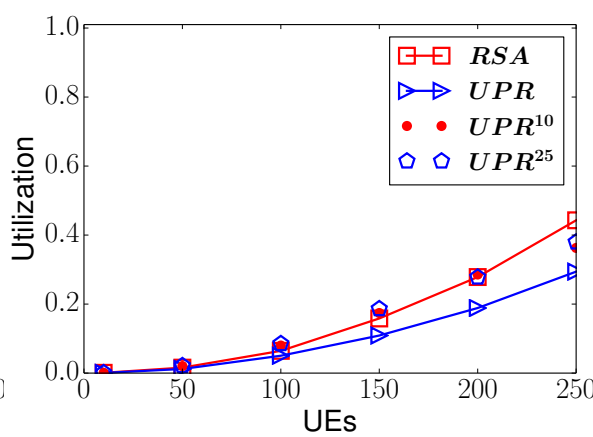

Fig. 5. Utilization of Band-b for RSA and UPRs.

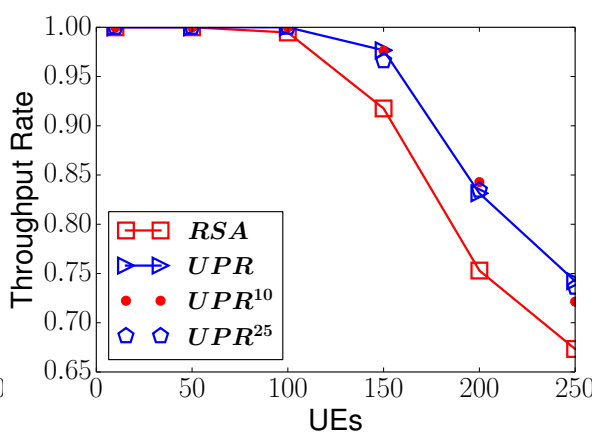

Fig. 8. RT traffic throughput for RSA and UPRs.

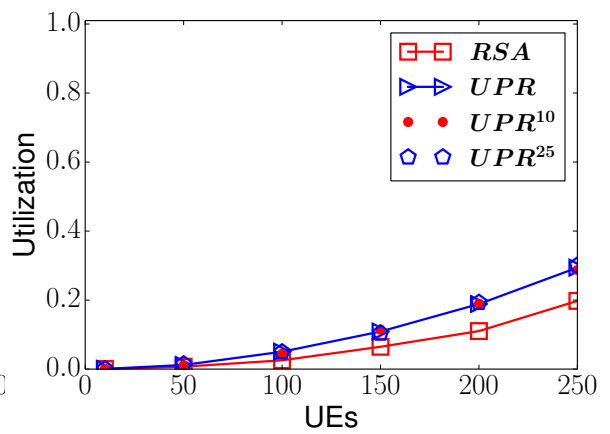

Fig. 6. Utilization of Band-c for RSA and UPRs

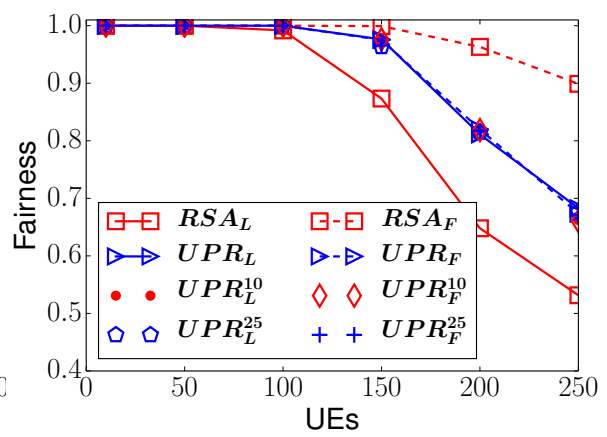

Fig. 9. Fairness: Device base throughput rate for RSA and UPRs. but estimated data usage of the UE can be $125 \mathrm{MB}$ or $75 \mathrm{MB}$ for $\mathrm{UPR}^{25}$ and 110 or 90 for $\mathrm{UPR}^{10}$. Therefore, the proposed method is evaluated under more realistic scenario.

\section{Packet Scheduling}

Without packet scheduling, the result cannot be obtained. Therefore, we have used a simple packet scheduling method in order to compare RSA and UPRs (UPRs represents UPR, $\mathrm{UPR}^{10}$ and $\mathrm{UPR}^{25}$ together). Packet arrival traffics are kept same for RSA and UPRs. UPRs dynamically arrange the number of CCs based on user profiles and maximum possible number of CCs is used for RSA. For test case, predetermined static number of CCs is four for RSA because maximum number of CCs for each UE is five in LTE systems and one of them must be used for PCC (see Section II). Because of UEs and eNB positions, CQI for all CCs is same for RSA and UPRs during the simulation. RSA and UPRs transfer each packet by using one of assigned CCs. If there are multiple packets arrived from a UE, RSA and UPRs may transfer packets over one or more of available CCs (without exceeding the number of CCs) based on device types. If there are multiple available $\mathrm{CCs}$ from different bands, firstly CCs which belongs to lower range band $(B a n d-c)$ are preferred to transfer the packet in order to decrease traffic loads to higher range band (Band-a).

\section{RESULts}

In this section, we present the performance of RSA and UPRs by comparing utilization of bands, throughput of NRT and RT traffics and fairness of service. Utilization of bands is measured by dividing total packets of active users in each CC to total capacity of CCs in each band then averaging the result with total time steps (simulation time/10ms). Throughput rates are measured by dividing transferred packets to all generated packets for NRT and RT. Therefore, while increasing number of UEs, throughput of traffic decreases for each UE. Fairness of service is calculated based on throughput rate of UEs type in order to see whether the service is provided fairly to all device types. By these comparisons, resource usage and managed QoS can be compared. The method which has lower resource usage and higher throughput with equal fair service between device types is better.

\section{A. Utilization}

Figs. 4, 5 and 6 show the utilization for Band-a, Band-b and Band-c, respectively, obtained for RSA and UPRs. If utilization of Band-a, Band- $b$ and Band-c are compared, it is observed that while the number of UEs is getting higher, utilization of all bands is gradually increasing for all cases. However, utilization of Band- $a$ is increasing faster than utilization of Band- $b$, utilization of Band- $b$ is increasing faster than utilization of Band-c for all cases. There are three reasons for it: (i) bands which have higher range are used more than bands which have lower range, (ii) distribution of UEs around the eNB increases probability of lower amount of UEs located in bands which have lower range and vice verse, and (iii) CCs assignment based on R method without considering CCs loads.

Bands utilization results of UPRs are close to each other for all bands while even error rate is increased to $\% 10$ and $\% 25$. 
Second interesting result is that utilization of UPRs in Band-c is higher than RSA and it vice verse in Band-a.

\section{B. Throughput Rate}

Figs. 7 and 8 show throughput rates of NRT and RT traffics. While the number of UEs is increased, throughput rate per UE is decreasing for both traffic type. While user profile has error rate up to \%25, throughput rates of UPRs are almost equal and higher than RSA for NRT and RT traffics. However, When the number of UEs is reached to 250, NRT throughput are almost equal for all cases.

\section{Fairness}

Fig. 9 show the service fairness between device types. $*_{L}$ represents LTE and LTE-A-Low capacity devices while $*_{F}$ represents LTE-A full capacity devices. By using RSA, LTEA full capacity devices get more service than LTE and LTE-A low capacity devices. However, UPRs are capable to fairly distribute service to UEs.

\section{Summary of Results}

Based on the results, we make the following observations: (i) while $\mathrm{R}$ method is simple, its performance is low in terms of throughput rate and utilization when the number of UEs is high. Therefore, increasing the number of users eventually results in less throughput rate, (ii) UPRs show improved (almost 20\%) throughput performance comparing to RSA, (iii) although increasing error percentage of user profile can be expected to significantly affects the overall throughput in $\mathrm{R}$ systems, up to $25 \%$ error does not decrease performance of user profile CCs assignment method, and (vi) higher bandwidth size of bands increases traffic throughput because RT traffic throughput is higher than NRT traffic for $\mathrm{R}$ methods.

\section{CONCLUSION}

In this paper, we have proposed a carrier component assignment method for LTE and LTE-A systems by considering user profiles. Throughput of non-real time and real time traffic, and bands utilization have been compared through extensive simulations. Results show that the proposed method uses system resources efficiently and provides improved user throughput rate and utilization in LTE and LTE-A systems. Our proposed method and related analysis will help service providers build efficient LTE-A systems architectures which are adaptable to LTE and LTE-A type devices by considering user profile, traffics and bands performances, such as throughput and utilization.

\section{REFERENCES}

[1] F. Richter. (2013, Sep.) Smartphone sales break the billion barrier. Accessed: June. 12, 2014. [Online]. Available: http://www.statista.com/ chart/777/global-connected-device-shipments/

[2] H. T. Dinh, C. Lee, D. Niyato, and P. Wang, "A survey of mobile cloud computing: architecture, applications, and approaches," Wireless Communications and Mobile Computing, Oct. 2011.
[3] H. Singh, J. Hsu, L. Verma, S. S. Lee, and C. Ngo, "Green operation of multi-band wireless LAN in $60 \mathrm{GHz}$ and $2.4 / 5 \mathrm{GHz}$," in Consumer Communications and Networking Conference $(C C N C)$, Las Vegas, NV, Jan 9-12, 2011, pp. 787-792.

[4] J. Wannstrom. (2013, June) LTE-Advanced. [Online]. Available: http://www.3gpp.org/technologies/keywords-acronyms/97-lte-advanced

[5] I. F. Akyildiz, D. M. Gutierrez-Estevez, and E. C. Reyes, "The evolution to 4G cellular systems: LTE-Advanced," Physical Communication, vol. 3, pp. 217-244, March 2010.

[6] L. xiang Lin, Y. an Liu, F. Liu, G. Xie, K. ming Liu, and X. yang $\mathrm{Ge}$, "Resource scheduling in downlink LTE-Advanced system with carrier aggregation," The Journal of China Universities of Posts and Telecommunications, vol. 19, no. 1, pp. 44 - 49, Feb. 2012.

[7] N. Kolehmainen, J. Puttonen, P. Kela, T. Ristaniemi, T. Henttonen, and M. Moisio, "Channel quality indication reporting schemes for UTRAN long term evolution downlink," in IEEE Vehicular Technology Conference, Singapore, May 11-14 2008, pp. 2522-2526.

[8] S.-B. Lee, S. Choudhury, A. Khoshnevis, S. Xu, and S. Lu, "Downlink MIMO with frequency-domain packet scheduling for 3GPP LTE," in INFOCOM, Rio de Janeiro, Apr. 19-25 2009, pp. 1269-1277.

[9] S. Donthi and N. Mehta, "Performance analysis of subband-level channel quality indicator feedback scheme of LTE," in National Conference on Communications, Chennai, Jan. 29-31 2010.

[10] H. Yang, F. Ren, C. Lin, and J. Zhang, "Frequency-domain packet scheduling for 3GPP LTE uplink," in INFOCOM, San Diego, CA, Mar. 14-19 2010.

[11] Y. Wang, K. Pedersen, T. Sorensen, and P. Mogensen, "Carrier load balancing and packet scheduling for multi-carrier systems," IEEE Transactions on Wireless Communications, vol. 9, no. 5, pp. 1780-1789, May 2010.

[12] X. Chen, H. Yi, H. Luo, H. Yu, and H. Wang, "A novel CQI calculation scheme in LTE $\backslash$ LTE-A systems," in International Conference on Wireless Communications and Signal Processing, Nanjing, Nov. 9-11 2011.

[13] F. Liu, W. Xiang, Y. Zhang, K. Zheng, and H. Zhao, "A novel QoEbased carrier scheduling scheme in LTE-Advanced networks with multiservice," in Vehicular Technology Conference, Quebec City, Canada, Sept. 3-6 2012.

[14] H. K. Rath, M. Sengupta, and A. Simha, "Novel transport layer aware uplink scheduling scheme for LTE-based networks," in National Conference on Communications, New Delhi, India, Feb. 15-17 2013.

[15] S. Bodas, S. Shakkottai, L. Ying, and R. Srikant, "Scheduling for small delay in multi-rate multi-channel wireless networks," in INFOCOM, Shanghai, China, Apr. 10-15 2011.

[16] W. Fu, Q. Kong, W. Tian, C. Wang, and L. Ma, "A QoS-aware scheduling algorithm based on service type for LTE downlink," in International Conference on Computer Science and Electronics Engineering, Hangzhou, China, Mar. 22-23 2013, pp. 2468-2474.

[17] R. Sivaraj, A. Pande, K. Zeng, K. Govindan, and P. Mohapatra, "Edgeprioritized channel- and traffic-aware uplink carrier aggregation in LTEAdvanced systems," in International Symposium on a World of Wireless, Mobile and Multimedia Networks, San Francisco, CA, June 25-28 2012.

[18] T. Girici, C. Zhu, J. R. Agre, and A. Ephremides, "Proportional fair scheduling algorithm in OFDMA-based wireless systems with QoS constraints," Journal of Communications and Networks, vol. 12, pp. 3042, 2010.

[19] X. Cheng, G. Gupta, and P. Mohapatra, "Joint carrier aggregation and packet scheduling in LTE-Advanced networks," in Communications Society Conference on Sensor, Mesh and Ad Hoc Communications and Networks, New Orleans, LA, June 24-27 2013, pp. 469-477.

[20] T. Dean and P. Fleming, "Trunking efficiency in multi-carrier CDMA systems," in 56th Vehicular Technology Conference,, vol. 1, Vancouver, Canada, Sep. 24-28 2002, pp. 156-160 vol.1.

[21] Ixia. (2013, Dec.) Quality of service (QoS) and policy management in mobile data networks. White Paper, Accessed: July. 10, 2014. [Online]. Available: http://www.ixiacom.com/pdfs/library/white_papers/ policy_management.pdf

[22] E. Perahia, C. Cordeiro, M. Park, and L. L. Yang, "IEEE 802.11ad: Defining the next generation multi-Gbps Wi-Fi," in 7th IEEE Consumer Communications and Networking Conference (CCNC), Las Vegas, NV, Jan 9-12, 2010. 